\title{
ON THE EQUATORIAL ACCELERATION OF THE SUN
}

\author{
IAN W. ROXBURGH \\ Queen Mary College, University of London, England
}

\begin{abstract}
The interaction of rotation and turbulent convection gives rise to a latitude dependent turbulent energy transport. Energy conservation demands a slow meridional circulation in the solar outer convective zone. The transport of angular momentum by this circulation is balanced in a steady state by the turbulent viscous transport across an angular velocity gradient. Models are constructed which give equatorial acceleration as observed on the sun.
\end{abstract}

\section{Fundamental Equations}

In this paper we shall consider as simple a model as possible that still retains the fundamental points of the theory. We are, after all, interested primarily in understanding the phenomenon of equatorial acceleration; the full quantitative treatment will be very complex and is postponed to a later date. We shall, for example, ignore the energy carried by radiation within the convective zone. This is satisfactory over most of it but obviously breaks down at the boundary where convective energy transport ceases and all the energy is carried by radiation. With this approximation we write the turbulent energy flux as

$$
\mathbf{F}=k \nabla S
$$

where

$$
S=c_{\mathrm{v}} \ln \left(P / \varrho^{\gamma}\right)
$$

is the entropy, $P$ the pressure and $\varrho$ the density. We shall throughout ignore the effects of ionisation and take $\gamma=\frac{5}{3}$. The 'turbulent conduction' $k$ varies from place to place, being a function both of depth and latitude.

In the energy balance equation we neglect viscous dissipation; if we thought it worthwhile this effect could be included, but it would only alter the quantitative not the qualitative result, so we shall neglect it here. With the 'conductivity' $k$ varying with latitude the divergence of the turbulent energy flux will not in general be zero and there will be a general circulation also carrying energy. Energy balance is then expressed by

$$
\nabla \cdot F+\frac{3}{2} P \mathbf{v} \cdot \nabla S=0 .
$$

The convective zone is assumed to be a viscous layer so that in a steady state it satisfies the equation

$$
\nabla \frac{V^{2}}{2}-\mathbf{v} \operatorname{curl} \mathbf{v}+\frac{\nabla P}{\varrho}+\nabla \Phi+\frac{1}{\varrho} \mathbf{R}=0
$$

where $\mathbf{v}$ is the total velocity, the sum of the circulation velocity $\mathbf{v}_{\mathbf{m}}$ and the rotation. $\Phi$ is the gravitational potential and $\mathbf{R}$ the viscous force. 
The turbulent viscous force in a convective layer is a notoriously difficult thing to estimate. We shall here assume that it has the same form as kinematic viscosity with the coefficient of viscosity $\eta=\varrho v_{\mathrm{t}} l$ where $\varrho$ is the density, $v_{\mathrm{t}}$ the mean turbulent velocity and $l$ the mixing length. The azimuthal $(\phi)$ component of the viscous force is then:

$$
R_{\phi}=-\frac{1}{r^{3}} \sin \theta \frac{\partial}{\partial r}\left(r^{4} \eta \frac{\partial \Omega}{\partial r}\right)-\frac{1}{r \sin ^{2} \theta} \frac{\partial}{\partial \theta}\left(\sin ^{3} \theta \eta \frac{\partial \Omega}{\partial \theta}\right) .
$$

In an axially symmetric situation this viscous force balances the convection of angular momentum by the thermally driven circulation $\mathbf{v}$, hence

$$
-\frac{\varrho}{r \sin \theta}\left[v_{\mathrm{r}} \frac{\partial}{\partial r}\left(\Omega r^{2} \sin ^{2} \theta\right)+\frac{v_{\theta}}{r} \frac{\partial}{\partial \theta}\left(\Omega r^{2} \sin ^{2} \theta\right)\right]=R_{\phi} .
$$

If $\mathbf{v}_{\boldsymbol{\theta}}$ is known this equation determines $\Omega(r, \theta)$.

EXAMPLE

As an example we shall consider the case where

$$
k=k(r, \theta)=K_{0}(r)\left(1+\varepsilon(r) P_{2}(\cos \theta)\right)
$$

and $\varepsilon \ll 1$. Using a series expansion in $\varepsilon$, gives

$$
\frac{k_{0}}{r^{2}} \frac{\mathrm{d}}{\mathrm{d} r}\left(r^{2} \frac{\mathrm{d} S_{0}}{\mathrm{~d} r}\right)+\frac{\mathrm{d} k_{0}}{\mathrm{~d} r} \frac{\mathrm{d} S_{0}}{\mathrm{~d} r}=0
$$

for the zero-order state, and

$$
\nabla P_{0} / \varrho=-\nabla \phi_{0} .
$$

If we further assume that $v$ is always so small as to be negligible in the hydrostatic balance the terms of order $\varepsilon$ yield

$$
v_{r}=\frac{\mathrm{d} \varepsilon}{\mathrm{d} r}\left(\frac{2 k_{0}}{3 P}\right) P_{2}=\frac{\eta \mathrm{d} \varepsilon}{\varrho} \frac{\mathrm{d} r}{2} P_{2}
$$

since $2 k / 3 \varrho=\eta$ in the simple mixing length theory. Hence the stream lines of $v$ are given by $S(r, \theta)=\psi(r) \sin \theta P_{2}^{\prime}$ so that

$$
v_{\mathrm{r}}=\frac{6}{r^{2} \varrho} \psi P_{2}, \quad v_{\theta}=-\frac{1}{r \varrho} \frac{\mathrm{d} \psi}{\mathrm{d} r} P_{2}^{\prime} .
$$

If we now turn to the equation that determines $\Omega$ we note that for $\eta$ large the solution is uniform rotation $\Omega=\Omega_{0}=$ constant. For large but finite $\eta$ we write $\Omega=\Omega_{0}+\omega_{1}(r)$ $+\omega_{2}(r) P_{2}$ and find

$$
\frac{1}{r^{3}} \frac{\mathrm{d}}{\mathrm{d} r}\left(r^{4} \eta \frac{\mathrm{d} \omega_{2}}{\mathrm{~d} r}\right)-\frac{10 \omega_{2} \eta}{r}=-4 \Omega_{0} r^{2} \frac{\mathrm{d}}{\mathrm{d} r}\left(\frac{\eta}{6 r} \frac{\mathrm{d} \varepsilon}{\mathrm{d} r}\right) .
$$

The solution is obviously independent of the magnitude of $\eta$, but does depend on ts variation in space. The solution depends on the interaction of rotation and con- 
vection through $\varepsilon(r)$. To simplify the numerical work we shall introduce variables.

so that

$$
s=\eta / \eta_{0}, \quad y=\omega_{2} / \varepsilon_{0} \Omega_{0}, \quad x=r / R, \quad \varepsilon=\varepsilon_{0} f
$$

$$
\frac{1}{x^{3}} \frac{\mathrm{d}}{\mathrm{d} x}\left(x^{4} s \frac{\mathrm{d} y}{\mathrm{~d} x}\right)-\frac{10 y s}{x}=-\frac{2}{3} x^{2} \frac{\mathrm{d}}{\mathrm{d} x}\left(\frac{s \mathrm{~d} f / \mathrm{d} x}{x}\right)
$$

subject to the free surface boundary conditions $\mathrm{d} y / \mathrm{d} x=0$ at $x=1, x_{\mathrm{i}}$, where the convective zone extends from $r=R$ to $r=r_{\mathrm{i}}=x_{\mathrm{i}} R$.

PARTICULAR CASE $s=1, \mathrm{~d} \varepsilon / \mathrm{d} x=1$

This is a highly idealised case but is sufficient to give some insight into the nature of the solution. $y$ satisfies the equation

$$
\mathrm{d}^{2} y+\frac{4 \mathrm{~d} y}{x}-\frac{10 y}{\mathrm{~d}^{2} x}=\frac{2}{3} x
$$

which has the solution

$$
y=\frac{1}{18} \frac{\left(1-x_{\mathrm{i}}^{6}\right) x^{2}}{\left(1-x_{\mathrm{i}}^{7}\right)}-\frac{x_{\mathrm{i}}^{6}\left(1-x_{\mathrm{i}}\right)}{45\left(1-x_{\mathrm{i}}^{7}\right) x^{5}}-\frac{x}{9} .
$$

For $x_{\mathrm{i}}=0.8$, an approximate solar model, this gives $y_{\mathrm{s}}=-0.06$. So that applying our model to the sun gives

$$
\frac{\Omega_{\mathrm{eq}}}{\Omega_{\mathrm{pole}}}=\frac{\left(1-\omega_{2 / 2} / \Omega_{0}\right)}{1+\omega_{2} / \Omega_{0}}=1-3 \omega_{2} / 2=1+\varepsilon_{0} 0.09,
$$

that is an equatorial acceleration.

FURTHER WORK

The problem and solution given above is in very simplified form. Before any definite conclusion may be reached a detailed model including both radiative and convective energy transport must be calculated. This is being done by Dr. Durney and myself. Already one detailed solution has been obtained and we expect to publish details of this work in the future. 